\title{
THE SEMICELLULARITY THEOREM ${ }^{1}$
}

BY

GENE G. GARZA

\begin{abstract}
In this paper are proved several theorems concerning semicellularity of subsets of 2-spheres in $E^{3}$. In particular, it is shown that a cellular arc or disk on a 2-sphere which has no nonpiercing points is semicellular in both complementary domains of the 2-sphere. The proof is entirely geometrical and involves the idea of piercing points.
\end{abstract}

I. Introduction. This paper answers the following question (Theorem 3.6) in the affirmative: Is a cellular disk $D$ which is a subset of a 2-sphere semicellular in both complementary domains of $\Sigma$ if $D$ has no nonpiercing points of $\Sigma$ ?

Most of the necessary definitions are standard and can be found in [7] or in [8]. The notation and abbreviations are also fairly standard, for example, $d$ will represent the usual 3-space metric. The standard techniques of cutting and pasting will be used as will simple geometric linking arguments [8, p. 285]. Also, the concept of "pushing by isotopy" will be used. An adequate reference is p. 80 of [21].

II. Semicellularity. Main theorem and corollary. We shall prove the following theorem in this section.

THEOREM 2.1. Suppose $\Sigma$ is a 2-sphere in $E^{3}$ which is locally flat $\bmod$ a cellular arc $\alpha$ with no nonpiercing points of $\Sigma$. Then $\alpha$ is semicellular in both complementary domains of $\Sigma$.

However, before starting the proof, we shall give a few definitions.

Definition 2.1. A set $K$ in $E^{3}$ is cellular if there exists a sequence $\left\{C_{i}\right\}$ of 3-cells in $E^{3}$ such that $C_{i+1} \subset$ Int $C_{i}$ and $\cap_{i=1}^{\infty} C_{i}=K$.

Definition 2.2. If $S$ is a 2-sphere in $E^{3}$, then any topological space homeomorphic with $S \cup \operatorname{Int} S$ is called a crumpled cube.

Definition 2.3. If $C$ is a crumpled cube and $p$ is a point on $\mathrm{Bd} C$, then $p$ is a piercing point of $C$ if there exists a homeomorphism, $h: C \rightarrow S^{3}$ such that $h(\operatorname{Bd} C)$ can be pierced with a tame arc at $h(p)$, i.e., there exists a tame arc which intersects $h(\mathrm{Bd} C)$ in exactly one point and has its endpoints in different complementary domains of $h(\mathrm{Bd} C)$.

Received by the editors August 17, 1979 and, in revised form, February 16, 1981.

1980 Mathematics Subject Classification. Primary 57N45, 57N60; Secondary 57M30, 57N75.

${ }^{1}$ This paper was taken from the author's dissertation which was written under the direction of Professor James Cantrell. 
Definition 2.4. The penetration index of an arc $\beta$ at a point $x$ is the smallest number for which there exist arbitrarily small 2-spheres about $x$ each hitting $\beta$ in no more points than this number [1].

Definition 2.5. If $C$ is a crumpled cube and $K$ is a subset of $\mathrm{Bd} C$, then $K$ is semicellular in $C$ if for each open set $U$ in $C$ containing $K$ there is an open set $V$ such that $K \subset V \subset U$ and each loop in $V-K$ is nullhomotopic in $U-K$.

Note the similarity in the definition of semicellular and the following characterization of piercing points.

TheOREM 2.2 (MCMillan [18]). If $C$ is a crumpled cube and $p$ is a point on Bd $C$, then $p$ is a piercing point of $C$ if for each open set $U$ in $C$ containing $p$ there is an open set $V$ such that $\{p\} \subset V \subset U$ and each loop in $V-\{p\}$ is nullhomotopic in $U-\{p\}$.

In view of McMillan's theorem it is natural to ask if $\alpha / \alpha$ is a piercing point for $\Sigma / \alpha$ in $E^{3} / \alpha$, which is again $E^{3}$ since $\alpha$ is cellular, in Theorem 2.1 . If it were, this would of course be sufficient to prove the theorem since deleted neighborhoods $U-\{\alpha / \alpha\}, V-\{\alpha / \alpha\}$ would be homeomorphic to $U-\alpha$ and $V-\alpha$, respectively, under the quotient or projection map $\pi: E^{3} \rightarrow E^{3} / \alpha$. Thus Theorem 2.1 can be reduced to showing that $\Sigma / \alpha$ is flat in $E^{3} / \alpha$. It is known that $\Sigma / \alpha$ is flat in $E^{3} / \alpha$ from at least one side, i.e., $\Sigma / \alpha$ along with one complementary domain is known to be a 3-cell [9]. Furthermore, it is known that $\Sigma / \alpha$ is flat provided it is arcwise accessible at $\alpha / \alpha$ by a tame arc on both sides of $\Sigma / \alpha$ [15].

Thus $\Sigma / \alpha$ is flat in $E^{3} / \alpha$ if and only if $\alpha / \alpha$ is a piercing point of $\Sigma / \alpha$, and, if and only if $\alpha$ is semicellular in both complementary domains of $\Sigma$. By these known facts or explicitly in [11, p. 224] it follows that $\alpha$ is semicellular in one of the crumpled cubes defined by $\Sigma$, say $\overline{\text { Int } \Sigma}$. Since semicellularity is a topological property of $\alpha$ in either Int $\bar{\Sigma}$ or $\overline{\text { Ext } \Sigma}$, we 'change' $E^{3}$ to $S^{3}$ and embed $\overline{\text { Ext } \Sigma}$ in $S_{3}$ in such a manner that $\overline{\text { Int } \Sigma}$ is a cell. This is possible by the Hosay-Lininger Theorem [14, 16]. This arc $\alpha$ would remain a cellular arc after the re-embedding in $S^{3}$ since $\overline{\text { Int } \Sigma}$ is now a cellular 3-cell. That Int $\bar{\Sigma}$ is cellular follows from the fact that $\Sigma$ is weakly flat which follows either [22, p. 13] or [9]. That $\alpha$ is cellular then follows from [17, p. 150].

The problem remains for us to decide upon some easy criterion to use to determine if $\Sigma / \alpha$ is flat, or if $\alpha / \alpha$ is a piercing point for $\Sigma / \alpha$. Gillman provides most of the answer for us in [13]. There he shows that a piercing point of $\Sigma$ (or $\Sigma / \alpha$ ) can be characterized as any point lying on a tame arc on $\Sigma$ (or $\Sigma / \alpha$ ). Furthermore, if $\alpha / \alpha$ is a nonpiercing point, any arc on $\Sigma / \alpha$ passing through $\alpha / \alpha$ is wild. These arcs would, however, be tame except for one point. If this point is an endpoint we could check the tameness of the arc by simply showing the penetration index at this endpoint to be one [1]. In view of the above discussion, if $\alpha_{0}$ is a tame extension of $\alpha$ on $\Sigma$, it is sufficient to find 2 -spheres about $\alpha$ in arbitrary neighborhoods of $\alpha$ which intersect $\alpha_{0}$ in one point each.

We now reduce the proof of Theorem 2.1 to the following lemma. 
Lemma 2.1. Suppose $\Sigma$ is a 2-sphere in $S^{3}$ such that $\mathrm{Cl}(\operatorname{Int} \Sigma)$ is a 3-cell. Suppose $\Sigma$ is locally flat $\bmod a$ cellular arc $\alpha$ with no nonpiercing points of $\Sigma$. Suppose $p$ is an endpoint of $\alpha$ and each subarc of $\alpha$ not containing $p$ is semicellular in both complementary domains of $\Sigma$. Then $\alpha$ is semicellular in both complementary domains of $\Sigma$.

The reduction goes as follows: We first recall that Theorem 2.1 is a statement about the semicellularity of $\alpha$, and since $\alpha$ is known to be semicellular in at least one domain [11, p. 224], say Int $\Sigma$, we re-embed $\overline{\operatorname{Ext}} \Sigma$ so that Int $\bar{\Sigma}$ is a 3-cell [14, 16]. This, of course, changes $\Sigma$ but its image (still called $\Sigma$ ) is also locally flat mod a cellular arc (since it lies on a cellular 3-cell as mentioned earlier). Furthermore, $\Sigma$ has no nonpiercing points after this re-embedding since $\overline{\text { Ext } \Sigma}$ has none, and Int $\Sigma$ is a 3-cell and therefore, also has none [17].

Next, we parametrize $\alpha$ with the interval $[0,1]$ and let $\alpha_{0}$ and $\alpha_{1}$ be tame extensions of $\alpha$ at 0 and 1 respectively which we assume have diameters at least twice that of $\alpha$. Then either $\left[0, \frac{1}{3}\right]$ or $\left[\frac{2}{3}, 1\right]$ is semicellular in both domains of $\Sigma$ since cellular arcs on 2-spheres contain at most one nonpiercing point [19, p. 153]. Suppose $\left[0, \frac{1}{3}\right]$ is semicellular in both domains. A least upper bound argument shows that there is either a first point $p \in(0,1]$ such that $[0, p]$ is not semicellular or a last point $p$ such that $[0, p]$ is semicellular. In the first case we approximate $\Sigma-\left(\alpha_{0} \cup[0, p]\right)$ with a sphere locally $P L \bmod \alpha_{0} \cup[0, p]$ and then use the new sphere as $\Sigma$. In the second case we mod out $[0, p]$ and use $\alpha=[p, 1]$ (and $\Sigma=\Sigma /[0, p])$. This unification of cases is possible since, in the first case, $\alpha_{0}$ would project to a wild arc in $S^{3} /[0, p]$ which we will show cannot happen. In the second case, $\alpha_{1}$ would project to a wild arc in $S^{3} /[p, 1]$ (if $p \neq 1$ ) which cannot happen by the same proof that we shall give for case 1 . If $p=1$, we have essentially the same case as either of the previous cases, i.e., $\Sigma$ is locally flat mod a cellular arc with no nonpiercing points and all subarcs of $\alpha$ are semicellular (in both complementary domains of $\Sigma$ ) except maybe those which contain the endpoint $p$. This completes our reduction of Theorem 2.1 to Lemma 2.1.

Our proof of Lemma 2.1 is lengthy and involves many cut and paste arguments; the goal is simple, however. We wish to find a sphere $\bar{\Sigma}$ and a disk $H$ that satisfy the hypothesis of the following technical lemma.

Lemma 2.2. Suppose $\Sigma$ is a 2-sphere in $S^{3}$ that is locally flat $\bmod$ a cellular arc $\alpha$ in $\Sigma$. Suppose $\bar{\alpha}$ is a tame arc in $\Sigma$ such that $\alpha \cap \bar{\alpha}$ is an endpoint of each. Suppose for each $\varepsilon>0$ there exist a PL-2-sphere $\bar{\Sigma}$ and a PL disk $H$ such that

1. $\bar{\Sigma} \cup H \subset N_{\varepsilon}(\alpha)$,

2. $\bar{\alpha}$ pierces $\bar{\Sigma}$ exactly once and otherwise misses $\bar{\Sigma}$,

3. $\alpha \cap \bar{\alpha} \subset$ Int $\bar{\Sigma}$,

4. $H \cap(\alpha \cup \bar{\alpha})=\varnothing$, and

5. Bd bounds $a$ disk $\bar{H}$ in Int $\bar{\Sigma}$ such that Int $\bar{H} \cap$ Int $H=\varnothing$ and the 2-sphere $H \cup \bar{H}$ contains $\alpha \cap \bar{\Sigma}$ in its interior. Then there exists a 2-sphere $S$ such that $\alpha \subset$ Int $S \subset N_{e}(\alpha)$ and $\bar{\alpha}$ pierces $S$ exactly once. Furthermore, $\alpha$ is semicellular in both complementary domains of $\Sigma$. 
Proof. Cut and paste on $\bar{\Sigma} \cup H$ by replacing subdisks of $\bar{\Sigma}-\bar{\alpha}$ with subdisks of $H$ until $\bar{\Sigma}$ and $H$ are disjoint. This gives a new sphere $S$ which contains $\alpha$ in its interior and which intersects $\bar{\alpha}$ in only one point. That $S$ does not hit $\alpha$ is seen by using the fact that $\bar{\Sigma} \cap \bar{H}=\varnothing$. We do, however, still need to observe that $\alpha$ lies inside the new sphere $S$; but this is clear since $S \cap \alpha=\varnothing$ and $\bar{\alpha}$ pierces $S$ at $z$ as it $\operatorname{did} \bar{\Sigma}$. This shows that $S$ contains one of the endpoints of $\bar{\alpha}$ and since we may assume the second endpoint does not lie in $N_{e}(\alpha)$ while $S$ does, we may conclude that $0 \in$ Int $S$ and therefore $\alpha \in$ Int $S$. The fact that $\bar{\alpha} \cap S$ is a single point shows that $\alpha$ is semicellular in both complementary domains of $\Sigma$.

Now we are ready to begin the proof of Lemma 2.1.

Proof (of Lemma 2.1). Assume $\alpha_{0}$ and $\alpha_{1}$ (from the reduction) intersect in a single point so that $\alpha_{0} \cup \alpha \cup \alpha_{1} \equiv \alpha^{\prime}$ is a simple closed curve. Let $\left\{\Sigma_{n}\right\}$ be the boundaries of a defining sequence of 3-cells for the cellular $\operatorname{arc} \alpha$. Let $\left\{S_{i}\right\}$ be the boundaries of a defining sequence for $p=1$ with the property that $S_{i} \cap \alpha_{1}$ is a single point $s_{i}$ for each $i$ and each $S_{i}$ is tame. This is possible since $\alpha_{1}$ is tame and therefore has a penetration index of 1 at $p$ [1]. We shall further assume that $S_{i} \cap \Sigma$ is especially nice for each $i$.

Claim I. Each $S_{i}$ may be assumed to be the union of two tame disks $D_{0}^{i}$ and $D_{1}^{i}$ with the following five properties (which will be preserved later even though the $S_{i}$ 's may be changed somewhat).

(1) Int $D_{0}^{i} \subset$ Int $\Sigma$ and $D_{0}^{i} \cap \alpha$ is a single point $d^{i}$;

(2) $\mathrm{Bd} D_{0}^{i}=\operatorname{Bd} D_{1}^{i}$ is the only simple closed curve of $S_{i} \cap \Sigma$ to intersect $\alpha_{1}$;

(3) $D_{1}^{i} \cap \Sigma$ is the union of a null sequence of tame simple closed curves $\left\{C_{j}^{i}\right\}_{j=1}^{\infty}$ and a totally disconnected set $C^{i}$;

(4) all simple closed curves in $D_{1}^{i} \cap \sum$ intersect $\alpha$ (for each $i$ ) and $C^{i} \subset \alpha$; and

(5) the $S_{i}$ 's are pairwise disjoint and only one component of $\alpha-S_{i}-S_{j}, i \neq j$, intersects both spheres.

To obtain the $S_{i}$, we need only to choose a null sequence of tame simple closed curves on $\Sigma$ about $p$ which intersect $\alpha$ and $\alpha_{1}$ only once each. These are easily capped in Int $\Sigma$ with a null sequence of disks using the collar structure on $\Sigma$ in $\overline{\text { Int } \Sigma}$. The $D_{1}^{i}$ can then be easily obtained by capping these simple closed curves in Ext $\Sigma$. Of course, it may not be possible to cap totally within Ext $\Sigma$; but, by using Theorem 4.6.5 of [8, p. 284] (or Bing [4]), to approximate $\Sigma-\left(\alpha_{0} \cup \alpha_{1} \cup\right.$ $\left(\cup \mathrm{Bd} D_{1}^{i}\right)$ ) we may cap each Bd $D_{1}^{i}$ "above" the $D_{0}^{i}$ to form the $S_{i}$. Then Theorem 5.5.2 of [8, p. 297], (or Bing [4]) can be used to complete (2) and (3). For condition (4) we would simply eliminate any circles of intersection which do not intersect $\alpha^{\prime}$ by cutting and pasting. By choosing a subsequence, if necessary, we may assume that the $S_{i}$ 's are pairwise disjoint and that only one component of $\alpha-S_{i}-S_{j}$, $i \neq j$, intersects both spheres. This completes Claim $\mathrm{I}$.

We order the $S_{i}$ in the obvious manner, from larger to smaller and assume that $S_{1}$ is small enough that $0 \in \operatorname{Ext} S_{1}$. Let $q_{1}$ and $q_{1}^{\prime}$ denote the points in $\alpha \cap S_{1}$ with the property that $\left[q_{1}, 1\right] \cap S_{1}=q_{1}$ and $\left[0, q_{1}^{\prime}\right] \cap S_{1}=q_{1}^{\prime}$. Then a new sequence is chosen by choosing $S_{2}$ so that it intersects $\alpha$ inside $\left(q_{1}, 1\right)$ only, i.e., $\left[0, q_{1}\right] \subset$ Ext $S_{2}$. By induction we choose $q_{i}, q_{i}^{\prime}$ for all $i$. 
Let $\varepsilon>0$ be given and less than both $\operatorname{dist}\left(\alpha_{0} \cap \alpha_{1}, \alpha\right)$ and $\operatorname{diam}(\alpha)$. We will construct a 2-sphere within $\varepsilon$ of $\alpha$ which intersects either $\alpha_{0}$ or $\alpha_{1}$ only once which would actually be sufficient to show that $\alpha$ is thereby separated from $S^{3}-N_{e}(\alpha)$. To do this, let $A$ be a disk on $\Sigma$ which lies within $N_{e}(\alpha)$ and contains $\alpha$ in its interior. Choose yet another sequence if necessary so that $S_{1}$ is an $\varepsilon$-sphere within $N_{e}(\alpha)$ and $S_{1} \cap \Sigma \subset A$. Consider $S_{i}, i=1, \ldots, 6$. Choose $q \in\left(q_{6}, 1\right)$ and $\left\{\Sigma^{j}\right\}$, the boundaries of a defining sequence for $[0, q]$. Since $[0, q]$ is semicellular in both crumpled cubes (i.e., $[0, q] /[0, q]$ is a piercing point for $\Sigma /[0, q]$ in $S^{3} /[0, q]$ ), we may choose the defining sequence $\left\{\Sigma^{j}\right\}$ so that each element intersects $\alpha_{0}$ only once. Then $\Sigma^{1}$ is selected small enough for the following conditions to hold.

(1) $\Sigma^{1} \subset N_{\varepsilon}(\alpha)$;

(2) $\Sigma^{1} \cap \alpha^{\prime} \subset\left(q_{6}, 1\right)$ except for $\Sigma^{1} \cap \alpha_{0}=$ point $z$; and

(3) $\Sigma^{1} \cap \Sigma \subset A$.

Let $\left\{\Sigma_{n}\right\}$ be the boundaries of a defining sequence of 3-cells for the cellular arc $\alpha$. We next choose $N$ so large that, if $i \geqslant N, \Sigma_{i}$ satisfies several conditions-the first being: $\Sigma_{i}$ intersects $\alpha_{1}$ inside $S_{6}$ and $\alpha_{0}$ inside $\Sigma^{1}$. Secondly, $\Sigma_{i}(i>N)$ will be such that $\Sigma_{i} \subset N_{\varepsilon}(\alpha)$ and $\Sigma_{i} \cap \Sigma \subset A$. Let $\left\{B^{i}\right\}_{i=2}^{6}$ be disks on $\cup_{i=2}^{6} S_{i}$ with the following properties.

(1) Each $B^{i}$ is the union of two disks $B_{0}^{i}$ and $B_{1}^{i}$ where $B_{0}^{i} \subset D_{0}^{i}$ and $B_{1}^{i} \subset D_{1}^{i}$ and $B_{0}^{i} \cap \mathrm{Bd} D_{0}^{i}=B_{1}^{i} \cap \mathrm{Bd} D_{1}^{i}$;

(2) $\operatorname{Bd}\left(B_{1}^{i}\right)-B_{0}^{i} \subset$ Ext $\Sigma$;

(3) Int $B^{i}$ contains $d^{i}$; and

(4) $\left(\cup B^{i}\right) \subset$ Int $\Sigma^{1}$.

Note that this requires $\operatorname{Bd} B^{i} \cap \alpha=\varnothing$. Finally, $N$ is chosen so that for $j \geqslant N$, $\Sigma_{j} \cap D_{0}^{i} \subset B_{0}^{i}-\mathrm{Bd}^{i}$ and $\mathrm{Bd}^{i} \cap \Sigma_{j}=\varnothing, i=2, \ldots, 6$. We now make some adjustments so that the intersections $\Sigma_{N} \cap \Sigma$ and $\Sigma_{N} \cap S_{i}, i=2, \ldots, 6$, are especially nice.

Claim II. $\Sigma_{N} \cap \Sigma$ may be assumed to be a finite collection of disjoint simple closed curves which bound disjoint disks $E_{j}, j=1, \ldots, m$, on $\Sigma_{N}$ with Int $E_{j} \subset$ Int $\Sigma$ and which separate $\alpha$ from $\operatorname{Bd} A$, i.e., the disks on $A$ bounded by $\left\{\operatorname{Bd} E_{j}\right\}_{j=1}^{m}$ contain $\alpha$. Furthermore, these simple closed curves intersect $\alpha_{0} \cup \alpha_{1}$ and $\operatorname{Bd} D_{0}^{i}$, $i=2, \ldots, 6$, in two points each. Also, $S_{i} \cap E_{j}$ is a single arc $e_{i, j}$ for each $i$, $i=2, \ldots, 6$, and $j, j=1, \ldots, m$.

The first part is easily seen by observing that $\overline{\text { Int } \Sigma / \alpha}$ is a 3-cell in $S^{3} / \alpha$ which has at most one wild point. For the second part we use the collar structure, not just for $\overline{\text { Int } \Sigma}$ but on both sides of $\Sigma-\alpha$ [21, p. 83], to adjust $\Sigma_{N}$ so that the intersection of $\alpha_{0} \cup \alpha_{1}$ with each 1-sphere, $\mathrm{Bd} E_{j}$, is two points. This is done by considering the innermost 0-sphere in $\operatorname{Bd} E_{j} \cap\left(\alpha_{0} \cup \alpha_{1}\right)$. Let us call two points from $\mathrm{Bd} E_{j} \cap\left(\alpha_{0} \cup \alpha_{1}\right)$ adjacent or innermost on $\alpha_{0}, \alpha_{1}$ or $\mathrm{Bd} E_{j}$ if they bound an arc on $\alpha_{0}, \alpha_{1}$ or $\mathrm{Bd} E_{j}$, respectively, that contains no other points of $\mathrm{Bd} E_{j} \cap\left(\alpha_{0} \cup\right.$ $\left.\alpha_{1}\right)$. It is then clear that there exist 0 -spheres which are innermost on both of either (1) $\mathrm{Bd} E_{j}$ and $\alpha_{0}$ or (2) $\mathrm{Bd} E_{j}$ and $\alpha_{1}$ in the case that $\mathrm{Bd} E_{j} \cap\left(\alpha_{0} \cup \alpha_{1}\right)$ is more than two points. Suppose it is case (1). Let $A^{\prime}$ be the subdisk of $A$ bounded by the two arcs from $\operatorname{Bd} E_{j}$ and $\alpha_{0}$. Note that $A^{\prime} \subset A-\alpha$ and is therefore tame. Using a 
local collar on $\Sigma-\alpha$, we fatten up $A^{\prime}$ to $A^{\prime} \times\left[-\varepsilon^{\prime}, \varepsilon^{\prime}\right] \equiv A^{\prime \prime}$ with $A^{\prime} \equiv A^{\prime} \times\{0\}$ where $\varepsilon^{\prime}$ is some appropriately small number. We then consider $\overline{\text { Int } \Sigma_{N}} \cup A^{\prime \prime}$ or $\mathrm{Cl}\left(\overline{\operatorname{Int} \Sigma_{N}}-A^{\prime \prime}\right)$. If Int $A^{\prime} \subset \operatorname{Ext} \Sigma_{N}$, we use $\operatorname{Bd}\left(\overline{\operatorname{Int} \Sigma_{N}} \cup A^{\prime \prime}\right)$ as $\Sigma_{N}$ which has the property that Bd $E_{j}$ intersects $\alpha_{0} \cup \alpha \cup \alpha_{1}$ as before except now the intersection of $\operatorname{Bd} E_{j}$ and $\alpha_{0}$ contains an arc. This arc may be removed by general position, thereby reducing $\operatorname{Bd} E_{j} \cap \alpha_{0}$ by two points. If Int $A^{\prime} \subset$ Int $\Sigma_{N}$, we use $\mathrm{Bd} \mathrm{Cl}\left(\overline{\mathrm{Int}} \Sigma_{N}-A^{\prime \prime}\right)$ as $\Sigma_{N}$ which then must be adjusted by general position to eliminate arcs in $\mathrm{Bd} E_{j} \cap \alpha_{0}$. Analogously, we may do this for each simple closed curve $\operatorname{Bd} D_{0}^{i}$. Note that this can be done without changing the properties assigned to each $B^{i}$. We may likewise insure that each 1-sphere in $D_{1}^{i} \cap \Sigma$, except $\operatorname{Bd} D_{1}^{i}$, does not intersect any $\mathrm{Bd} E_{j}$. Then, using the collar structure on $\overline{\operatorname{Int} \Sigma}$ we may cap off in Int $\Sigma$, if necessary, the simple closed curves in $\cup_{i, j} C_{j}^{i}$, except for $\cup_{i=2} \mathrm{Bd} D_{1}^{i}$, whose disks (on $\cup D_{1}^{i}$ ) hit $\cup E_{j}$. Again, note that none of the five properties (see Claim I) of the $S_{i}$ have been changed and that they are still $\varepsilon$-spheres. Any simple closed curves in $\left(\cup E_{j}\right) \cap S_{i}$, i.e., $\left(\cup E_{j}\right) \cap D_{0}^{i}$, are eliminated by replacing subdisks on the $E_{j}$ by those on $D_{0}^{i}$ in the usual manner. The $\Sigma_{N}$ and $S_{i}$ resulting from these adjustments will be called the "originals". This completes Claim II.

Now we must adjust $\Sigma_{N} \cap\left(\cup_{i=2}^{6} S_{i}\right)$ in several special cases with all necessary adjustments being made within $N_{\varepsilon}(\alpha)$ in order to find $\bar{\Sigma}$ and $H$ of the technical lemma. We will assume that $\Sigma_{N} \cap\left(\cup_{2}^{6} S_{i}\right)$ is a finite collection of disjoint simple closed curves since each sphere is tame. Let $G \subset \Sigma_{N} \cap S_{4}$ be an innermost 1-sphere on $\Sigma_{N}$. Let $\Sigma_{N}(G)$ denote the innermost disk it bounds on $\Sigma_{N}$ and $S_{4}(G)$ the disk it bounds on $S_{4}-s_{4}$. (If $G$ is the only simple closed curve in $\Sigma_{N} \cap S_{4}$ then $\Sigma_{N}(G)$ may be either of the two disks $G$ bounds on $\Sigma_{N}$.) The adjustments are made in the following cases.

I. Int $\Sigma_{N}(G) \subset$ Int $S_{4}$.

A. $S_{4}(G) \cap \alpha=\varnothing$.

(1) $\Sigma_{N}(G) \cap \alpha_{1}=\varnothing$.

In this case we replace $\Sigma_{N}(G)$ with $S_{4}(G)$ and push off slightly (after pushing subdisks of $\Sigma_{N}$ off Int $S_{4}(G)$ ).

(2) $\Sigma_{N}(G) \cap \alpha_{1} \neq \varnothing$.

This case is essentially the same as I.A.(1), but we should note that after this adjustment, there might be simple closed curves in $\Sigma_{N} \cap \Sigma$ which no longer separate $\alpha$ from $\operatorname{Bd} A$. These are eliminated in the obvious manner by replacing subdisks of $\Sigma_{N}$ with subdisks of $\Sigma$.

Note. These adjustments can be made so close to $\Sigma$ that the thus-adjusted $\Sigma_{N}$ intersects only the original $S_{i}$, i.e., it does not need to intersect any of the disks previously used to adjust the $S_{i}$. Thus all simple closed curves of the adjusted intersection, $\Sigma_{N} \cap\left(\cup_{2}^{6} S_{i}\right)$, are 1-spheres that also lie on the original $S_{i}$.

B. $S_{4}(G) \cap \alpha \neq \varnothing$.

(1) $\Sigma_{N}(G) \cap \alpha_{1}=\varnothing$.

Note that in this case $\Sigma_{N}(G) \subset$ Ext $\Sigma$, since otherwise $\Sigma_{N}(G)$ would contain "half" of some $E_{j}$ and therefore intersect $\alpha_{1}$. By considering innermost simple closed curves in $\Sigma_{N}(G) \cap\left(S_{5}-s_{5} \cup S_{6}-s_{6}\right)$ and the disks they bound on $\Sigma_{N}(G)$ 
we may adjust $S_{5}$ and $S_{6}$ so that they remain disjoint. Of course, all of this takes place inside $S_{4}$. Then $S_{4}$ is adjusted by replacing $S_{4}(G)$ by $\Sigma_{N}(G)$ and pushing off once again. This can also be done so that $S_{4}$ does not intersect $S_{5}$ or $S_{6}$ afterward, and so that all other important properties of the $S_{i}$ are maintained.

(2) $\Sigma_{N}(G) \cap \alpha_{1} \neq \varnothing$.

Here we shall either adjust $\Sigma_{N}(G)$ so that it does (1) not intersect $\alpha_{1}$, or (2) intersects it only once, or (3) we shall find a special subdisk of it which hits $\alpha_{1}$ only once. In cases (2) and (3) we then use the disk obtained by this adjustment to play the role of $H$ in the technical lemma (2.1). $\Sigma^{1}$ will play the role of $\bar{\Sigma}$. Using the lemma we would then obtain a sphere about $\alpha$ in $N_{e}(\alpha)$ which intersects $\alpha_{0}$ only once. This in turn would be sufficient to show that $\alpha_{0}$ has a penetration index of one [1] in (and is therefore tame in) the decomposition space $S^{3} / \alpha$. This adjustment goes as follows.

Consider $S_{4}-s_{4}$ as a plane $P_{4}$ and note its intersection with $\Sigma$ and $\Sigma_{N}$. In $P_{4}$, Bd $D_{0}^{4}-s_{4}$ is a line $L^{4}$ which intersects $\alpha$ in a single point $d^{4}$. Let $L^{4}=L_{-}^{4} \cup L_{+}^{4}$ with $L_{-}^{4} \cap L_{+}^{4}=d^{4}$. Let $H_{+}^{4}$ and $H_{-}^{4}$ denote the upper and lower half-planes of $P_{4}$, respectively, so that $H_{-}^{4}=D_{0}^{4}-s_{4}$. Similarly, we write $L_{ \pm}^{5}, L_{ \pm}^{6}, H_{ \pm}^{5}, H_{ \pm}^{6}$, etc., where $L_{-}^{5}$ and $L_{-}^{6}$ are on the same side of $\alpha^{\prime}($ on $\Sigma)$ as $L_{-}^{4}$. Note that $\#\left(L_{-}^{4} \cap G\right)$ $=\#\left(L_{+}^{4} \cap G\right)$. This follows from the construction since any $e_{i, j}$ in $G$ has one endpoint in each of $L_{-}^{4}$ and $L_{+}^{4}$. If this number is 1 , we have the disk we need since each $E_{j}$ intersects $\alpha_{1}$ in only one point so that in fact $\#\left(L_{-}^{4} \cap G\right)=\#\left(\Sigma_{N}(G) \cap \alpha_{1}\right)$. So suppose this number is more than 1 . We will now either reduce this number by at least two or find the special disk mentioned earlier. This will then be repeated (with a new or adjusted $G$ ) until this number is zero, i.e., $G$ has been eliminated, or we find that disk.

Let $E_{4, j}$ denote the subdisk of $E_{j}$ which is also a subdisk of $\Sigma_{N(G)}$. Note that part of its boundary is $e_{4, j}$ and that it intersects $\alpha_{1}$. It is clear from the construction that if $E_{4, j} \cap \alpha_{1}$ is part of $\Sigma_{N}(G)$, then all of $E_{4, j}$ is. If we remove from $\Sigma_{N}(G)$ all the $E_{4, j}$ 's, we obtain a disk $Q$, since we are removing from $\Sigma_{N}(G)$ disjoint subdisks which each have a single arc of intersection with $G$. Consider the intersection of $S_{5}$ and $S_{6}$ with $Q$. Recall that each $E_{j}$ intersects $S_{5} \cup S_{6}$ only in $e_{5, j} \cup e_{6, j}$. We may thus assume that by cutting and pasting, $Q \cap\left(S_{5} \cup S_{6}\right)$ is a collection of arcs with endpoints in $L_{5} \cup L_{6}$. To see this, let $M$ be an innermost 1-sphere (on $Q$ ) in $Q \cap\left(S_{5} \cup S_{6}\right)$ and let $Q(M)$ be the disk it bounds on $Q$, then $Q(M) \cap \alpha_{1}=\varnothing$. This is the case since $Q(M) \subset \Sigma_{N(G)}-\cup E_{4, j}$ and since $\Sigma_{N}(G)$ is innermost on $\Sigma_{N} . M$ and all other simple closed curves in $Q \cap\left(S_{5} \cup S_{6}\right)$ are then eliminated by the usual cut and paste methods. Note once again that it is possible to do this and still keep $S_{5}$ and $S_{6}$ disjoint.

If there is an arc $\rho$ in $Q \cap\left(S_{5} \cup S_{6}\right)$ with both endpoints in some $E_{4, k}$ we have 'found' that special disk we wanted. It is the disk on $\Sigma_{N}(G)$ bounded by the circle in $\Sigma_{N}(G) \cap\left(S_{5} \cup S_{6}\right)$ which is formed by $e_{5, k}$ (or $\left.e_{6, k}\right)$ and $\rho$, since this disk can intersect $\alpha_{1}$ only once. So we assume for the moment that such a $\rho$ does not exist.

Let $\eta_{6}$ be an outermost arc of $Q \cap S_{6}$ on $Q$. Let $D_{\eta_{6}}$ be the outermost disk it determines on $Q$, that is, $\eta_{6}$ along with a subarc of $\mathrm{Bd} Q$, which has the same endpoints as $\eta_{6}$, form Bd $D_{\eta_{6}}$ and $D_{\eta_{6}} \cap S_{6}=\eta_{6}$. There are three cases to consider. 
(i) $\eta_{6}$ has both endpoints in $L_{-}^{6}$ or both in $L_{+}^{6}$.

In this case $D_{\eta_{6}} \subset \overline{\text { Ext } S_{6}}$. Probably the easiest way to see this is to observe that $\mathrm{Bd} Q$ is made up of subarcs of $G$ and subarcs of the boundaries of the $E_{4, j}$ and that these necessarily alternate. Further, from the construction those subarcs from the boundaries of the $E_{4, j}$ are intersected by $S_{6}$ in exactly two points (these points are the endpoints of the $e_{6, i}$ 's, one in $L_{+}^{6}$ and the other in $L_{-}^{6}$ ). Also from the construction $\alpha_{1}$ intersects each of these subarcs in one point which is between the two points where $S_{6}$ intersects. Therefore $D_{\eta_{6}}$ must be outside $S_{6}$.

From the above discussion, it is also clear that $S_{5} \cap D_{\eta_{6}}$ is a single arc $\eta_{5}$. And this arc along with the corresponding one, $l_{5}$, on $L^{5}$ bounds a disk $D\left(l_{5} \cup \eta_{5}\right)$ on $P_{5}$ which can be used to eliminate at least two 1-spheres from the intersection of $\Sigma_{N}$ and $\Sigma$, thus reducing $\sharp\left(L_{-}^{4} \cap G\right)$ by 2 or more. This can be accomplished in the following manner.

First, observe that $D\left(l_{5} \cup \eta_{5}\right) \cap \alpha=\varnothing$. To see this, note that $D_{\eta_{6}} \cap S_{4}$ is an arc $\eta_{4}$ with endpoints in the same two $E_{j}$ as $\eta_{6}$ by the construction. Let $l_{4}$ be an arc on $L^{4}$ corresponding to $\eta_{4}$ and $D\left(l_{4} \cup \eta_{4}\right)$ be the disk they bound on $P_{4}$. Let $l_{6}$ on $L_{6}$ correspond to $\eta_{6}$ and let $D\left(l_{6} \cup \eta_{6}\right)$ be the disk $l_{6} \cup \eta_{6}$ bounds on $P_{6}$. Then

$$
\operatorname{Bd}\left(D_{\eta_{6}} \cup D\left(l_{6} \cup \eta_{6}\right) \cup D\left(l_{4} \cup \eta_{4}\right)\right) \equiv K
$$

is a simple closed curve on $\Sigma$ which is composed of $l_{6} \cup l_{4}$ and two subarcs of the $E_{j}$ (i.e., their boundaries) which contain the endpoints of $l_{6}$. Int $D\left(l_{5} \cup \eta_{5}\right)$ lies inside the sphere formed by $Z \equiv D_{\eta_{6}} \cup D\left(l_{6} \cup \eta_{6}\right) \cup D\left(l_{4} \cup \eta_{4}\right) \cup \Sigma(K)$ where $\Sigma(K)$ is the usual small disk on $\Sigma$ bounded by $K$. This is the case, since we have already adjusted the intersection of $D_{\eta_{6}}$ and $S_{5}$ (recall that $D_{\eta_{6}} \subset Q$ ) so that there are no 1-spheres in the intersection; and since we have adjusted the intersection of $S_{5}$ and $\Sigma$ so that $S_{5}$ can intersect $\Sigma(K)$ only in $l_{5}$ (recall that $D_{1}^{5}$ does not intersect the $E_{4, j}$ 's, except in two boundary points each). Thus, $D\left(l_{5} \cup \eta_{5}\right) \cap \alpha=\varnothing$ since a component of $\alpha-\left(S_{4} \cup S_{5} \cup S_{6}\right)$ can intersect at most two of these spheres and since the component $\left(q_{4}, q_{6}^{\prime}\right)$ of $\alpha-\left(S_{4} \cup S_{6}\right)$ obviously lies outside $Z$. Now, by extending $D\left(l_{5} \cup \eta_{5}\right)$ into a slightly larger disk $F$ on $S_{5}$ with $F \cap \Sigma$ still only an arc, we may isotope $D\left(l_{5} \cup \eta_{5}\right)$ (and $\eta_{5}$ in particular) into Int $\Sigma$ with an isotopy on $F$ which has been extended to a small neighborhood of $F$. Let $E_{j_{1}}$ and $E_{j_{2}}$ be the $E_{j}$ which contain the endpoints of $l_{5}$. The isotopy would have changed the two 1-spheres, $\operatorname{Bd}\left(E_{j_{1}} \cup E_{j_{2}}\right)$, into a single simple closed curve which no longer separates $\alpha$ from $\mathrm{Bd} A$. This simple closed curve and any such others are eliminated from the thus-adjusted $\Sigma \cap \Sigma_{N}$ by usual methods. This reduces $\sharp\left(L_{-}^{4} \cap G\right)$ as planned.

(ii) $\eta_{6}$ has its endpoints in both $L_{-}^{6}$ and $L_{+}^{6}$ and Int $D_{\eta_{6}} \subset$ Int $S_{6}$.

To be outermost, $\eta_{6}$ would, in this case, have its endpoints in the same $E_{4, j}$ which would contradict our earlier assumption against the existence of $\rho$.

(iii) $\eta_{6}$ has its endpoints in both $L_{-}^{6}$ and $L_{+}^{6}$ and Int $D_{\eta_{6}} \subset$ Ext $S_{6}$.

$D_{\eta_{6}}$ is, of course, a disk whose boundary consists of two subarcs, $a$ and $b$, from two $E_{4, j}$ (i.e., their boundaries), $\eta_{6}$, and a subarc $\eta_{4}$ of $G . S_{5} \cap D_{\eta_{6}}$ is again a single $\operatorname{arc} \eta_{5}$. But now consider the other intersection arcs of $S_{5} \cap Q$. Since the endpoints of $\eta_{5}$ cannot lie in the same $E_{4, j}$ (by assumption), there must be an arc $\eta_{5}^{\prime}$ in $S_{5} \cap Q$ 
with both of its endpoints outside the endpoints of $\eta_{5}$ (on $L_{5}$ ) and with both in either $L_{-}^{5}$ or $L_{+}^{5}$. Then, as in (i), we may eliminate at least two simple closed curves from $\Sigma \cap \Sigma_{N}$ since the disk $D\left(l_{5}^{\prime} \cup \eta_{5}^{\prime}\right)$ defined in the obvious manner on $P_{5}$ does not intersect $\alpha$. To see this we will need to construct a sphere which contains $\left(q_{4}, q_{6}^{\prime}\right)$ in its interior and then show that $D\left(l_{5}^{\prime} \cup \eta_{5}^{\prime}\right)$ lies in the sphere's exterior. This would be sufficient since, by construction, $D\left(l_{5}^{\prime} \cup \eta_{5}^{\prime}\right)$, which is a subset of $S_{5}$, intersects $\alpha$ only in $\left(q_{4}, q_{6}^{\prime}\right)$. We will use the following notation.

(1) $E_{1}$ is the first $E_{j}$ among those meeting $Q$.

(2) $R_{1}$ is the subdisk of $E_{1}$ determined by $D_{4}^{0}$ and $D_{6}^{0}$.

(3) $R_{4}$ and $R_{6}$ are the subdisks of $D_{4}^{0}-s_{4}$ and $D_{6}^{0}-s_{6}$ (respectively) determined by $E_{1}$.

(4) $R_{2}$ and $R_{3}$ are the subdisks of $A$ which have for their boundaries, four subarcs of $L_{4}$ and $L_{6}$ and the four subarcs of $\operatorname{Bd}\left(R_{1} \cup D_{\eta_{6}}\right)$ which lie on $\Sigma$. (If there are only three subarcs of $\operatorname{Bd}\left(R_{1} \cup D_{\eta_{6}}\right)$ on $\Sigma$, we will only need one disk, say $R_{2}$. If $\operatorname{Bd}\left(R_{1} \cup D_{\eta_{6}}\right)$ has only two arcs on $\Sigma$, then our assumption on the nonexistence of the $\operatorname{arc} \rho$ will have been contradicted.)

(5) $P_{4}(1)$ will denote the disk on $P_{4}$ which has the union of $\eta_{4}$ and the arc on $L_{4}$ corresponding to $\eta_{4}$, for its boundary.

(6) $P_{6}(1)$ will be the corresponding disk on $P_{6}$ (using $\eta_{6}$ instead of $\eta_{4}$ ).

(7) $J$ will denote the sphere formed by the union of $R_{1}, R_{2}, R_{3}, R_{4}, R_{6}, P_{4}(1)$, $P_{6}(1)$ and $D_{\eta_{6^{\circ}}}$

We will need to show that $J$ is actually a sphere. First, note that $R_{1} \cup R_{4} \cup R_{6}$ is a disk since $R_{1} \cap\left(R_{4} \cup R_{6}\right)$ is two subarcs of $\mathrm{Bd} R_{1}$. Similarly, $R_{1} \cup R_{4} \cup R_{6} \cup$ $R_{2} \cup R_{3}$ is a disk. To see that $R_{1} \cup R_{4} \cup R_{6} \cup R_{2} \cup R_{3} \cup P_{4}(1)$ is a disk one should observe that $P_{4}(1) \cap\left(R_{1} \cup R_{4} \cup R_{2} \cup R_{3}\right)$ is a single boundary arc and that $P_{4}(1) \cap R_{6}=\varnothing$ since $R_{6} \subset S_{6}$ while $P_{4}(1) \subset S_{4}$. Likewise $R_{1} \cup R_{4} \cup R_{6} \cup$ $R_{2} \cup R_{3} \cup P_{4}(1) \cup P_{6}(1)$ is a disk. By construction, $D_{\eta_{6}}$ does not intersect $S_{4} \cup S_{6}$ except in $\eta_{4} \cup \eta_{6}$ and since it lies on $Q$, it does not intersect $R_{1}$ (except in the special case where $\operatorname{Bd}\left(R_{1} \cup D_{\eta_{6}}\right) \cap \Sigma$ is composed of three arcs, and in this case it does so in one of the arcs in $\mathrm{Bd} R_{1} \cap \Sigma$ ). It also only intersects $R_{2}$ and $R_{3}$ in one boundary arc each. Now, since

$$
\mathrm{Bd}\left(R_{1} \cup R_{4} \cup R_{6} \cup R_{2} \cup R_{3} \cup P_{4}(1) \cup P_{6}(1)\right)=\mathrm{Bd} D_{\eta_{6}}
$$

we can conclude that $J$ is a sphere.

It is clear that $\left(q_{4}, q_{6}^{\prime}\right) \subset$ Int $J$ (if Int $J$ is the 'small' complementary domain of $J)$. Also, $D\left(l_{5}^{\prime} \cup \eta_{5}^{\prime}\right) \cap J=\varnothing$ which can be seen by observing that $D\left(l_{5}^{\prime} \cup \eta_{5}^{\prime}\right)$ does not intersect $R_{1} \cup R_{4} \cup R_{6} \cup P_{4}(1) \cup P_{6}(1)$ (for obvious reasons). Likewise, $D\left(l_{5}^{\prime} \cup \eta_{5}^{\prime}\right)$ does not intersect either $R_{2}$ or $R_{3}$ since it does not intersect their boundaries and since any 1-sphere from the intersection inside $R_{2} \cup R_{3}$ could be (indeed, would have been) eliminated by cutting and pasting. $D\left(l_{5}^{\prime} \cup \eta_{5}^{\prime}\right) \cap D_{\eta_{6}}=$ $\varnothing$ since $Q \cap S_{5}$ is a collection of arcs and since $D_{\eta_{6}} \cap S_{5}$ is the single arc $\eta_{5}$. Thus $D\left(l_{5}^{\prime} \cup \eta_{5}^{\prime}\right) \cap J=\varnothing$ and since it is clear that $D\left(l_{5}^{\prime} \cup \eta_{5}^{\prime}\right) \subset$ Ext $J$ (by construction) we conclude that $D\left(l_{5}^{\prime} \cup \eta_{5}^{\prime}\right) \cap \alpha=\varnothing$.

Note. As in case I.A.(2), these adjustments which are the same as those in I.A.(2) can be made so that the new circles in $\Sigma_{N} \cap\left(\cup_{2}^{6} S_{i}\right)$ also lie on the original $S_{i}$. 
Further, we should note that all adjustments are made without hitting the boundaries of the $B^{i}$ since these adjustments are made with subdisks of $\Sigma_{N}, A$ and with subdisks of the $B^{i}$, which do not hit $\mathrm{Bd} B^{i}, i=2, \ldots, 6$.

II. Int $\Sigma_{N}(G) \subset$ Ext $S_{4}$.

Using $S_{2}$ and $S_{3}$, this case is essentially the same as case I.

We may briefly summarize all of this as follows.

(1) Choose $G$ and $\Sigma_{N}(G)$.

(2) Decide whether Int $\Sigma_{N}(G)$ is in Int $S_{4}$ or Ext $S_{4}$.

(3) Make adjustments to $\Sigma_{N}(G)$.

(4) Choose new innermost $G$ and new $\Sigma_{N}(G)$.

(5) Continue until $H$ is obtained.

The proof of Lemma 2.1 will be complete when we show that the hypothesis of the technical lemma (2.2) holds. Conditions (1)-(4) are clear and so we need only to check (5). (The existence of $H$ is clear since we cannot eliminate all of the simple closed curves in $\Sigma_{N} \cap \Sigma$.) Let $\bar{\Sigma}$ be either $\Sigma^{1}$ or $S_{1}$, as the case may be, and let $\bar{H}$ be $P(\mathrm{Bd} H)$ where $P(\mathrm{Bd} H)$ is the disk on the original $\cup_{i=2}^{6} P_{i}$ bounded by $\mathrm{Bd} H$. That $H \cup \bar{H}$ contains $\alpha \cap \bar{\Sigma}$ in its interior is seen by observing that $\Sigma^{1}$ (or $\left.S_{1}\right) \cap P(\operatorname{Bd} H)=\varnothing$ since $P(\operatorname{Bd} H) \subset \cup_{i=2}^{6} B_{i} \subset$ Int $\Sigma^{1}$ (or Int $\left.S_{1}\right)$.

Thus, we have shown $\alpha$ to be semicellular in Ext $\Sigma$ since $\alpha_{0}$ and $\alpha_{1}$ project to tame arcs in $E^{3} / \alpha$. This, of course, proves the technical lemma, Lemma 2.1 and Theorem 2.1, since, as noted before, this property is independent of the embedding.

The following corollary follows very easily as pointed out to me by Bob Daverman.

COROLlary 2.1. Suppose $\Sigma$ is a 2-sphere in $S^{3}$ which is locally flat $\bmod$ a cellular disk $D$ with no nonpiercing points. Then $D$ is semicellular in both complementary domains of $\Sigma$.

Proof. $D$ can be squeezed to an arc $\alpha$ so that Theorem 2.1 applies [10, p. 87].

III. Consequences of the semicellularity theorem. In this section we will answer five of the questions asked by Burgess at the 1974 Utah Conference [7, pp. 53-55] and then give some further consequences of Theorem 2.1. Some of these questions were asked earlier by Daverman and Eaton in [11, p. 226]. However, the genesis of these problems seems to go back at least to Loveland [17, p. 151]. Loveland showed arcs and disks on the boundary of cellular 3-cells to be cellular and this was used in Theorem 2.1 and will be used in the following ones which could almost be listed as corollaries to Theorem 2.1.

THEOREM 3.1. Each disk in the boundary of a crumpled cube $C$ is semicellular in $C$ if Int $C$ is an open 3-cell and each point of $\mathrm{Bd} C$ is a piercing point of $C$.

Proof. Let $D$ be a subdisk of Bd $C \equiv \Sigma$. Embed $C$ in $S^{3}$ so that Ext $C$ is a ball $[14,16]$. Note that each point of $\Sigma$ is a piercing point after this re-embedding and that $D$ is now cellular. Let $\alpha$ be a tame arc on $\Sigma$ with only one endpoint hitting $D$ in Bd $D[13$, p. 465]. Approximate $\Sigma-D-\alpha$ so that it is locally flat. This gives a new sphere $\Sigma^{\prime}$ which is weakly flat since it is locally flat mod a cellular disk $D$ [22, 
p. 13]. The sphere $\Sigma^{\prime}$ likewise has no nonpiercing points [18]. Now we note that $D$ is semicellular in each complementary domain of $\Sigma^{\prime}$ by Corollary 2.1. Thus $\Sigma^{\prime} / D$ is flat in $S^{3} / D$ so that $\alpha$ projects to a tame arc in $S^{3}$. But this implies that $D / D$ is a piercing point for $\Sigma / D$ also. And as discussed before this implies $D$ is a semicellular in each complementary domain of $\Sigma$ which is sufficient to show that $D$ is semicellular in $C$ before the re-embedding.

THEOREM 3.2. If $D$ is a disk on a weakly flat 2-sphere $\Sigma$ and contains at most one nonpiercing point of $\Sigma$, then $D$ is cellular.

Proof. It is sufficient to show that $D$ is semicellular in one of the crumpled cubes of $\Sigma$ by [7, p. 51]. Let $C$ be the crumpled cube which has no nonpiercing point on $D$. If $C$ has a nonpiercing point, it is known that $D$ is semicellular in $C$ [7, p. 54]. But if $C$ has only piercing points, Theorem 3.1 shows $D$ is semicellular in $C$.

THEOREM 3.3. If $D$ is a disk in the boundary of a crumpled cube $C$ and every proper subdisk of $D$ is semicellular and Int $C$ is an open 3-cell, then $D$ is semicellular in $C$.

Proof. $C$ has at most one nonpiercing point $[6$, p. 325] and this point is not in $D$ since each point of a semicellular set is a piercing point for $C[18, \mathrm{p}$. 313]. Thus $D$ is semicellular in $C$ by Theorem 3.1.

THEOREM 3.4. Suppose $h: \Delta \times I \rightarrow E^{3}$ is an embedding such that:

(1) $h(\{x\} \times I)$ is a tame arc for each $x \in \Delta$;

(2) the disks $h(\Delta \times\{0\})$ and $h(\Delta \times\{1\})$ are cellular in $E^{3}$;

(3) the annulus $h(\mathrm{Bd} \Delta \times I)$ is tame in $E^{3}$;

(4) each of $h($ Int $\Delta \times\{0\})$ and $h($ Int $\Delta \times\{1\})$ can be pierced with a tame arc at each point.

Then the 3-cell $h(\Delta \times I)$ is cellular in $E^{3}$.

Proof. Bd $h(\Delta \times I)$ is locally flat mod two cellular disks with no nonpiercing points so it is weakly flat by Theorem 3.1 since $\mathrm{Bd} h(\Delta \times 1) / h(\Delta \times\{0,1\})$ has no nonpiercing points and is therefore flat. Thus each domain of $\operatorname{Bd} h(\Delta \times I)$ is an open 3-cell (use $S^{3}$ ) and $h(\Delta \times I)$ is cellular.

The following theorem is closely related to a question asked by Stewart [23] at the 1967 Arizona Conference. Stewart asked whether or not a 2-sphere would be weakly flat, i.e., both domains being open 3-cells, if all subdisks were cellular. In light of the counterexamples for Stewart's orginal question this seems to be the best possible result that one might obtain.

Theorem 3.5. Suppose $\Sigma$ is a 2-sphere in $E^{3}$ with the property that $\Sigma$ can be pierced by an annulus $A \times[-1,1]$ at some simple closed curve $A$. Suppose further that the two disks $D$ and $E$ bounded by $A$ are cellular and that $\Sigma$ has no nonpiercing points. Then $\Sigma$ is weakly flat.

Proof. Let $A \times[-1,1] \cap \Sigma=A \times\{0\} \equiv A$. By Bing's approximation theorem [3] we may assume $A \times[-1,1]$ is locally flat $\bmod A$. Let $\beta$ be a tame arc in $E$ with one point in $A$. Approximate Int $E-\beta$ so that it is locally flat. Then all of Int $E$ is 
locally flat, i.e., after the approximation [13]. By Theorem $3.1, \beta$ is tame in $E^{3} / D$ so that there exists a disk $D^{\prime}$ which forms a 'cap' for $D$ in say, $\overline{\operatorname{Int} \Sigma^{\prime}}$ which intersects $\Sigma^{\prime}$ only in its boundary and $\beta$ in only one point where $\Sigma^{\prime}$ is $\Sigma$ after the adjustment $E^{\prime}$ is made to $E$. This cap is easily constructed by considering $\overline{\text { Int } \Sigma^{\prime} / D}$. This is a 3-cell and the existence of 'caps' for $D / D$ is clear. Assume general position between $A \times[-1,1]$ and $D^{\prime}$ and suppose $A \times\{1\}$ lies in Int $\Sigma$ (and Int $\Sigma^{\prime}$ if we approximate $E-\beta$ closely enough to miss $\left.A \times[-1,1]\right)$. The set $D \cap A \times[0,1]$ is then a finite collection of disjoint circles. We remove the inessential circles (i.e., inessential on $A \times[0,1]-D / D$ ) by replacing subdisks of $D^{\prime}$ with subdisks of $A \times[0,1]$. Note that if $D^{\prime}$ is chosen close enough to $D$, these subdisks are also close to $D$. (The $\varepsilon-\delta$ argument is obvious.) Thus we assume the intersection has only essential circles. Let $\gamma$ be an innermost intersection circle on $D^{\prime}$. Let $D^{\prime}$. Let $D^{\prime}(\gamma)$ be the disk on $D^{\prime}$ bounded by $\gamma . D^{\prime}(\gamma) \cap \Sigma=\varnothing$ if we choose $D^{\prime}$ close enough to $D$. To see this consider the following.

$A \times[0,1] / D$ is locally flat $\bmod D / D=p$ and is a disk $F$ in $E^{3} / D . \beta$ is tame in $E^{3} / D$ and intersects $F$ at one point. Thus $F$ is flat on the other side [2, 9]. Using this, $F$ lies on a sphere $G$ which intersects $\Sigma / D$ only in $p . D^{\prime}(\gamma)$ can then easily be chosen to lie inside $G$.

Similarly, there exists an $E^{\prime}(\gamma)$ for $E . E^{\prime}(\gamma) \cup D^{\prime}(\gamma) \cup$ (the annulus on $A \times$ $[0,1]$ bounded by $\left.\operatorname{Bd}\left(E^{\prime}(\gamma) \cup D^{\prime}(\gamma)\right)\right)$ then forms a sphere in a small neighborhood of $\Sigma$ that does not intersect $\Sigma$ by construction. This can be done in either complementary domain. Thus from the construction and by Brown's Monotone Union Theorem [5], $\Sigma$ is weakly flat.

Theorem 3.6 represents what might be considered a mild generalization of Theorem 2.1 although its proof follows easily from 2.1. So far we have shown that cellular disks or arcs which lie on weakly flat spheres are semicellular disks in each complementary domain if they contain no nonpiercing points of the sphere. A natural question to ask is whether or not we need the hypothesis that the sphere be weakly flat. The answer is, as one might expect, "no". To see this we need only approximate the complement of the disk, say $D$, (or arc) on $\Sigma$, in such a manner that the new sphere is locally flat mod $D$ and $D$ contains no nonpiercing points for the approximation. This can easily be done by choosing a triangulation for $\Sigma-D$ such that the mesh converges to zero rapidly at $D$. This triangulation should, of course, be chosen in such a manner that the 1-skeleton, $\Delta^{1}$, consists entirely of tame arcs, and such that its closure on $\Sigma$ is obtained by adding $\operatorname{Bd} D$ if $D$ is a disk or $D$ if $D$ is an arc. We may then approximate $\overline{\Delta^{1 c}}$. The sphere, $\Sigma^{\prime}$, obtained is locally flat $\bmod D$, and since each point of $\operatorname{Bd} D$ lies on a tame arc $\beta$, say, in $\overline{\Delta^{1}}$ and since each point of Int $D$ is a piercing point for $D$ we may conclude that $D$ contains no nonpiercing points of $\Sigma^{\prime}$. Thus $\beta$ is tame in $E^{3} / D$. But $\beta$ lies on $\Sigma$ so that $D / D$ is a piercing point for $\Sigma$ and therefore $D$ is semicellular in both domains of $\Sigma$. This proves the following theorem.

THEOREM 3.6. Suppose $D$ is a cellular disk or arc on a 2-sphere $\Sigma$ in $S^{3}$ and $D$ contains no nonpiercing points of $\Sigma$. Then $D$ is semicellular in both complementary domains of $\Sigma$. 
We need this obvious extension of Theorem 2.1 in the following theorem.

THEOREM 3.7. Let $\Sigma$ be a 2-sphere in $S^{3}$ which has no nonpiercing points. Let $G$ represent a decomposition of $S^{3}$ which yields $S^{3}$ and whose only nondegenerate elements consist of cellular arcs or disks on $\Sigma$. Then each point $p$ of $\Sigma / G$ in $S^{3} / G$ is a piercing point.

Proof. $\Sigma / G$ is a sphere by Moore [20]. So it is sufficient to show that small tame curves about $p$ on $\Sigma / G$ can be shrunk missing $p$ in small neighborhoods of $p$ in either crumbled cube $K$ of $\Sigma / G$ by [18, p. 316]. But these curves are homotopic in $K-\{p\}$ to small loops in Int $K$ which pull back to loops in neighborhoods of $\pi^{-1}(p)$ in $S^{3}$ where $\pi: S^{3} \rightarrow S^{3} / G$ is the projection map. Since $\pi$ is uniformly continuous we may choose these loops so $\alpha$, say, and $\pi^{-1}(\alpha)$ lie sufficiently close to $p$ and $\pi^{-1}(p)$ so that we may use the semicellularity of $\pi^{-1}(p)$ to shrink $\pi^{-1}(\alpha)$ inside a small neighborhood of $\pi^{-1}(p)$ but missing $\pi^{-1}(p)$. The image of this singular disk is then projected into $K$ so that $p$ is piercing point for $K$. Thus $p$ is a piercing point for $\Sigma / G$ since $K$ was arbitrary.

An application of Theorem 3.7 results when considering whether or not a 2-sphere $\Sigma$ might be weakly flat if it is locally flat mod some fairly nice collection of cellular arcs or disks. Stewart showed that this is the case if the collection consisted of only one disk or arc. Theorem 2.1 easily extends this to any finite collection if there are no nonpiercing points on $\Sigma$. Theorem 3.7, however, shows that this is the case if, for example, the set $(G)$ of arcs and disks is a countably infinite upper semicontinuous collection with only a finite collection of accumulation points in $E^{3} / G$. This would follow from the fact that a disk which is locally flat mod one point $p$ which is a piercing point is flat. This follows in much the same manner as the case for a sphere which is locally flat mod one piercing point since such disks are known to lie on spheres [2].

The following is a list of propositions which follow easily from Theorem 2.1 and some questions whose answers might be determined by Theorem 2.1.

PROPOSITION 3.1. If $\alpha$ and $\beta$ are two cellular arcs that lie on a 2-sphere $\Sigma$ with no nonpiercing points and if $\alpha \cap \beta$ is an endpoint of each and if their union pierces a disk at $\alpha \cap \beta$, then $\alpha \cup \beta$ is cellular.

Proposition 3.2. Suppose $\alpha$ is an arc on $\Sigma$ with the property that all subarcs not containing an endpoint, say, $p$, are semicellular in both crumpled cubes of $\Sigma$ and that $p$ is a piercing point of $\Sigma$. Then $\alpha$ is cellular if there exists a sequence of points on $\alpha$ converging to $p$ at which $\alpha$ pierces disks. Furthermore, all subarcs of $\alpha$ are semicellular.

Proof. The proof is precisely the same as that of Theorem 2.1.

Proposition 3.3. Let $D$ be a disk which has a tame boundary and is locally flat mod a cellular subset. If $D$ lies on a 2-sphere $\Sigma$ and contains no nonpiercing points of $\Sigma$, then $D$ is cellular. Furthermore, $D$ could have any finite number of such cellular subsets. 
Question 3.1. Suppose $\Sigma$ is a 2-sphere in $S^{3}$ that is locally flat mod a cellular disk $D$ with one nonpiercing point $p$. Then $\Sigma / D$ has a nonpiercing point. Is the penetration index for an arc on $\Sigma / D$ with $D / D$ for one endpoint the same as that for an arc on $\Sigma$ which is locally flat mod one endpoint?

Question 3.2. Suppose $\alpha$ is an arc on a 2 -sphere $\Sigma$ in $S^{3}$ with the property that all subarcs not containing an endpoint, say $p$, are semicellular in both crumpled cubes of $\Sigma$ and that $p$ is a piercing point for $\Sigma$. Is $\alpha$ cellular?

Question 3.3. Is the one-point-union of two cellular arcs on $\Sigma$ cellular if they contain no nonpiercing points of $\Sigma$ ? (By one-point-union, I mean simply their union if their intersection is a single point.)

Note that the solution to this question would follow immediately to an affirmative answer to Question 3.2.

\section{BIBLIOGRAPHY}

1. W. R. Alford and B. J. Ball, Some almost polyhedral wild arcs, Duke Math. J. 30 (1963), 33-38.

2. Ralph J. Bean, Disks in $E^{3}$. I. Subsets of disks having neighborhoods lying on 2-spheres, Trans. Amer. Math. Soc. 112 (1964), 206-213.

3. R. H. Bing, Improving the side approximation theorem, Trans. Amer. Math. Soc. 116 (1965), 511-525.

4. Improving the intersections of lines and surfaces, Michigan Math. J. 14 (1967), 155-159.

5. Morton Brown, The monotone union of open n-cells, Proc. Amer. Math. Soc. 12 (1961), 812-814.

6. C. E. Burgess, Criteria for a 2-sphere in $S^{3}$ to be tame modulo two points, Michigan Math. J. 14 (1967), 321-330.

7. , Semicellular sets in crumpled cubes, Proc. 1974 Utah Geometric Topology Conf., Springer-Verlag, New York, 1975, pp. 42-57.

8. C. E. Burgess and J. W. Cannon, Embeddings of surfaces in $E^{3}$, Rocky Mountain J. Math. 1 (1971), 259-344.

9. J. C. Cantrell, Almost locally polyhedral 2-spheres in $S^{n}$, Duke Math. J. 30 (1963), 249-252.

10. R. J. Daverman, On cells in Euclidean space that cannot be squeezed, Rocky Mountain J. Math. 5 (1975), 87-93.

11. R. J. Daverman and W. T. Eaton, Universal crumpled cubes, Topology 11 (1972), 223-235.

12. P. H. Doyle and J. G. Hocking, Some results on tame disks and spheres in $E^{3}$, Proc. Amer. Math. Soc. 11 (1960), 832-836.

13. D. S. Gillman, Side approximation, missing an arc, Amer. J. Math. 85 (1963), 459-476.

14. Norman Hosay, Conditions for tameness of a 2-sphere which is locally tame modulo a tame set, Notices Amer. Math. Soc. 9 (1962), 117. Abstract \#589-43.

15. S. Kinoshita, On quasi translations in 3-space, Topology of 3-Manifolds, 1961 University of Georgia Topology Proc., Prentice-Hall, Englewood Cliffs, N. J., 1962, pp. 223-226.

16. L. L. Lininger, Some results on crumpled cubes, Trans. Amer. Math. Soc. 118 (1965), 534-549.

17. L. D. Loveland, Piercing points of crumpled cubes, Trans. Amer. Math. Soc. 43 (1969), 145-152.

18. D. R. McMillan, Some topological properties of piercing points, Pacific J. Math. 22 (1967), 313-322. 19. Piercing a disk on a cellular set, Proc. Amer. Math. Soc. 19 (1968), 135-157.

20. R. L. Moore, Concerning upper semi-continuous collections of continua, Trans. Amer. Math. Soc. 27 (1925), 416-428.

21. T. B. Rushing, Topological embeddings, Academic Press, New York and London, 1973.

22. D. G. Stewart, Cellular subsets of the 3-spheres, Trans. Amer. Math. Soc. 114 (1965), 10-22.

23. , On unions of cellular sets, Proc. Topology Conf., (Arizona State University, 1967),

Arizona State Univ., Tempe, 1968, pp. 262-264.

Department of Mathematics, University of Georgia, Athens, Georgia 30602

Current Address: Department of Mathematics, Louisiana State University, Baton Rouge, Louisiana 70810 